\title{
BMJ Open Body mass index trajectory from childhood to puberty and high blood pressure: the China Health and Nutrition Survey
}

\author{
Yiman Ji (D) , ${ }^{1,2}$ Xiangjuan Zhao, ${ }^{3}$ Yiping Feng, ${ }^{1,2}$ Yanlin Qu, ${ }^{1,2}$ Ying Liu, ${ }^{1,2}$ Sijia Wu, ${ }^{1,2}$ \\ Yutong Wu, ${ }^{1,2}$ Fuzhong Xue, ${ }^{1,2}$ Yunxia Liu (D) ${ }^{1,2}$
}

To cite: Ji Y, Zhao X, Feng Y, et al. Body mass index trajectory from childhood to puberty and high blood pressure: the China Health and Nutrition Survey. BMJ Open 2021;11:e055099. doi:10.1136/ bmjopen-2021-055099

- Prepublication history and additional supplemental material for this paper are available online. To view these files, please visit the journal online (http://dx.doi.org/10.1136/ bmjopen-2021-055099).

Received 06 July 2021 Accepted 27 October 2021

Check for updates

(C) Author(s) (or their employer(s)) 2021. Re-use permitted under CC BY-NC. No commercial re-use. See rights and permissions. Published by BMJ.

${ }^{1}$ Department of Biostatistics, School of Public Health, Shandong University Cheeloo College of Medicine, Jinan, Shandong, China

${ }^{2}$ Institute for Medical Dataology, Shandong University Cheeloo College of Medicine, Jinan, Shandong, China

${ }^{3}$ Department of gynaecology, Maternal and Child Health Care of Shandong Province, Shandong University Cheeloo College of Medicine, Jinan, Shandong, China

Correspondence to Professor Yunxia Liu; yunxialiu@163.com

\section{ABSTRACT}

Objectives The prevalence of childhood hypertension

is rising in parallel with the increasing prevalence of overweight and obesity in children. How growth trajectories from childhood to puberty relate to high blood pressure (HBP) is not well defined. We aimed to characterise potential body mass index (BMI) dynamic changing trajectories from childhood to puberty and investigate their association with HBP.

Design A dynamic prospective cohort.

Setting China Health and Nutrition Survey 1991-2015. Participants There were 1907 participants (1027 men and 880 women) in this study.

Outcomes The primary outcome was HBP defined as systolic blood pressure (SBP)/diastolic blood pressure (DBP) exceeding the standards or diagnosis by medical records or taking antihypertensive medication.

Results A model of cubic parameters with three groups was chosen, labelled as normal increasing group $(85.16 \%$, $\mathrm{n}=1624)$, high increasing group $(9.81 \%, \mathrm{n}=187)$ and resolving group $(5.03 \%, n=96)$. Compared with the normal increasing group, the unadjusted $\mathrm{HRs}(95 \% \mathrm{Cls})$ for the resolving and high increasing groups were 0.91 (0.45 to 1.86 ) and 1.88 (1.26 to 2.81), respectively. After adjusting for baseline age, region, sex, baseline BMI z-score, baseline SBP and baseline DBP in model 3, the HRs $(95 \%$ $\mathrm{Cls})$ for the resolving and high increasing groups were 0.66 (0.30 to 1.45$)$ and 1.56 (1.02 to 2.38).

Conclusions These results indicate that the BMI trajectories from childhood to puberty have significant impact on HBP risk. Puberty is a crucial period for the development of HBP.

\section{INTRODUCTION}

There is a high prevalence of hypertension in China ${ }^{1}$ and it is considered to be the leading risk factor for cardiovascular mortality. Worse still, the current prevalence of hypertension in children is estimated at about $1 \%-5 \%$, which is higher among minority adolescents. ${ }^{3}$ The prevalence of childhood hypertension is rising in parallel with the increasing prevalence of overweight and obesity in children. ${ }^{4}$ Many studies have shown
Strengths and limitations of this study

- A new lifetime approach (the latent class growth mixed model) was used to identify different trajectory patterns of body mass index (BMI) from childhood to puberty (5-19 years).

- This study also explored the relationships between the BMI trajectory groups and systolic blood pressure /diastolic blood pressure.

- This study fitted changes in nutrient intake, which is the important risk factor of high blood pressure, as well as the trends in height and weight of the participants. $^{9}$

- The lack of adjustment for biological maturation may affect the estimation of the impact of BMI trajectories on blood pressure.

that children with overweight or obesity have a significantly higher risk of hypertension than non-overweight children. ${ }^{5-8}$ However, the great majority of studies on this topic focused on body mass index (BMI) in a single or limited number of time points, ignoring the dynamic BMI changes that occur over time in child-to-puberty.

BMI trajectories can provide information of the potential patterns of BMI varying with age and allow a more accurate interpretation of the relationship between high blood pressure (HBP) and obesity. There are several studies focusing on identifying diverse BMI trajectories in childhood. ${ }^{9-14}$ Buscot et al ${ }^{15}$ identified six distinct BMI trajectories from early childhood to adulthood and their association with adult cardiometabolic risk. But few studies have focused on BMI trajectories from childhood to adolescence and linked these trajectories to the risk of HBP.

By using repeated measurements of BMI measured 3-6 times during 1991-2015 from the China Health and Nutrition Survey (CHNS), the current study aimed to identify 
potential BMI dynamic changing trajectories from childhood to puberty (5-19 years) and to examine the association of BMI trajectories with HBP.

\section{METHODS}

\section{Study cohort}

In this study, we used data from the CHNS, an ongoing open longitudinal cohort designed to investigate health and nutritional status in Chinese population during China's socioeconomic transition. ${ }^{16} 17$ The survey took place using a multistage, random cluster process to draw a sample of about 7200 households with over 30000 individuals. In addition, three mega cities have joined this cohort since 2011 and three more provinces joined since 2015. Ten cross-sectional surveys were undertaken between 1989 and 2015.

We excluded individuals with missing BMI information, less than three follow-up visits, prevalent hypertension at baseline, younger than 5 years old or older than 19 years old. In addition, we also excluded individuals with 4 SDs higher or lower than the average of height, weight or BMI. A total of 1907 subjects were included in this study and they were repeatedly visited 3-6 times. The average follow-up year was 7.3 years ( $5-14$ years). BMI data after the onset of HBP (outcome) were excluded from analyses. Online supplemental figure 1 shows the study population selection process. Online supplemental table 1 lists excluded respondents $(\mathrm{n}=9784)$ who were more likely to be women, generally older, with higher baseline BMI, higher baseline systolic blood pressure (SBP)/diastolic blood pressure (DBP), and more likely to be in urban.

\section{Examinations}

In each cross-sectional survey, standing height was measured without shoes to the nearest $0.1 \mathrm{~cm}$ using a portable SECA stadiometer (SECA, Hamburg, Germany). Weight was measured to the nearest $0.1 \mathrm{~kg}$ on a special scale that was calibrated routinely in light clothing without shoes. BMI was calculated as weight $(\mathrm{kg})$ divided by height $(\mathrm{m})$ squared. SBP and DBP were measured using a mercury sphygmomanometer in a sitting position after a 15 min rest. Each subject was measured three times and averaged.

The definition of HBP varied by age. For subjects older than 18 years, HBP was defined as SBP/DBP $\geq 140 / 90$ $\mathrm{mm} \mathrm{Hg}$ or diagnosis by medical records or taking antihypertensive medication. For those under the age of 18, we calculated the diagnostic criteria for HBP in children and adolescents based on the simplified formula criteria for hypertension screening according to 2018 Chinese guidelines for the management of hypertension. ${ }^{18}$ In addition, medical history or taking antihypertensive medication were used to assist judgement.

\section{Statistical methods}

To improve the comparability of BMI among children of different ages, we calculated individual BMI z-scores based on age-specific averages in the cohort. ${ }^{19}$ To obtain z-scores, raw BMIs were converted by subtracting the sample mean BMI from the individual BMI and dividing by sample SD at each age point. ${ }^{2021}$ The same conversion was done for height and weight to rule out outliers who were 4 SDs higher or lower than the mean based on z-scores.

The latent class growth mixed model (LCGMM) was used to identify different trajectory patterns of BMI z-score. ${ }^{22}$ Parameters of non-linear growth curves of BMI z-score were estimated using a random-effects mixed model as a function of age (centered to 12.21 years, the mean age of the cohort). In the modeling process, we used gender as a covariable to adjust for pattern differences between genders. The mixed model allows for repeated measurements of study variables and different number of individual observations at unequally intervals. The mixed model includes fixed and random effects representing deviations of BMI z-score for individuals from the fixedeffects parameters. The model computes maximum likelihood estimates of growth curve parameters, including fixed-effects parameters (for a group) and random-effects parameters (for each individual within the group). By combining these two types of parameters, 1907 different sets of growth curve parameters were generated for each participant in the study cohort with the intercept, linear and non-linear parameters varying from individual to individual.

The model selection was determined by the following criteria $^{22}$ : a reduction of Bayesian information criterion of at least 20; high mean posterior class membership probabilities $(>0.65)$; high mean posterior probabilities $(>0.7)$. Age and its higher order terms were included one by one for model building. The higher order terms of age were not included in the model, if they were not significant, or made lower order terms not significant or did not improve the goodness-of-fit of the model.

To compare characteristics between multiple different groups, Mann-Whitney test and Kruskal-Wallis test were used for continuous variables, and $\chi^{2}$ test was used for categorical variables. Cox proportional hazard model was used to investigate the association between the BMI trajectory groups and HBP with unadjusted in model 1, adjusted for baseline age, sex, region and baseline BMI z-score in model 2, adjusted for baseline age, sex, region, baseline BMI z-score and baseline SBP/DBP in model 3. Linear regression was also used to analyse the associations between BMI trajectory groups and SBP/DBP, and $\beta$ with SE was estimated. In addition to adjusting the covariates mentioned in Cox proportional hazard model, the linear regression also adjusted the variable of follow-up years in model 2 and model 3. All statistical analyses were conducted using R V.4.0.3. The statistical significance level was set at $\mathrm{p}<0.05$.

\section{RESULTS}

Table 1 summarises the baseline and follow-up characteristics by blood pressure status at follow-up. A total of 179 cases of HBP were identified during the follow-up period, with an 
Table 1 Baseline and follow-up characteristics by blood pressure status at follow-up

\begin{tabular}{|c|c|c|c|c|}
\hline Variable & $\begin{array}{l}\text { Total } \\
(n=1907)\end{array}$ & $\begin{array}{l}\text { Normal blood pressure } \\
(n=1728)\end{array}$ & $\begin{array}{l}\text { High blood pressure } \\
(n=179)\end{array}$ & $P$ value \\
\hline \multicolumn{5}{|l|}{ Baseline } \\
\hline Male, n (\%) & 1027 (53.8) & $928(53.7)$ & 99 (55.3) & 0.682 \\
\hline Urban, n (\%) & $506(26.5)$ & $460(26.6)$ & $46(25.6)$ & 0.790 \\
\hline BMI, $\mathrm{kg} / \mathrm{m}^{2}$ & $15.8(2.1)$ & $15.8(2.1)$ & $15.8(2.1)$ & 0.977 \\
\hline SBP, $\mathrm{mm} \mathrm{Hg}^{\star}$ & $89.1(10.3)$ & $88.8(10.2)$ & $91.3(10.3)$ & 0.006 \\
\hline DBP, mm Hg* & $58.1(8.3)$ & $57.9(8.3)$ & $60.0(8.0)$ & 0.002 \\
\hline \multicolumn{5}{|l|}{ Follow-up } \\
\hline Age, years & $16.0(2.0)$ & $16.1(2.0)$ & $14.6(1.8)$ & $<0.001$ \\
\hline $\mathrm{DBP}, \mathrm{mm} \mathrm{Hg}{ }^{*}$ & $69.7(8.8)$ & $68.3(7.9)$ & $82.6(5.5)$ & $<0.001$ \\
\hline Follow-up, years & $7.3(1.8)$ & $7.4(1.8)$ & $6.6(1.4)$ & $<0.001$ \\
\hline
\end{tabular}

Data are means (SD), or $\mathrm{n}(\%)$.

$P$ values: the differences between normal blood pressure group and high blood pressure group.

${ }^{*}$ Results of SBP/DBP were calculated after removing missing value

$\mathrm{BMI}$, body mass index; DBP, diastolic blood pressure; SBP, systolic blood pressure.

incidence density of 12.8 per 1000 person-years. Compared with the normal blood pressure group, participants in HBP group were younger and had higher baseline SBP, higher baseline DBP and longer follow-up years. These differences remained statistically significant until the end of the follow-up. In addition, participants in the HBP group were more likely to be men and live in urban than those in the normal blood pressure group and had higher BMI levels, although these differences were not statistically significant. Online supplemental table 2 shows the age distribution of onset in HBP group by gender. Online supplemental table 3 shows the baseline and follow-up survey years by blood pressure status at follow-up.

We fitted models from 1 class to 4 classes of linear, quadratic and cubic curves, respectively. A model of cubic parameters with three groups was chosen with lower Bayesian information criterion, higher posterior probabilities and group membership probability. The detailed information on the LCGMM results of fitting process is shown in online supplemental table 4 .

Figure 1 shows the three distinct trajectories of BMI, labelled as normal increasing group $(85.16 \%, n=1624)$, high increasing group $(9.81 \%, \mathrm{n}=187)$ and resolving group $(5.03 \%, \mathrm{n}=96)$. In the normal increasing group, BMI remained a low level within normal weight status throughout follow-up. In the high increasing group, BMI increased gradually, reaching a peak around age 16 and exceeding the upper limit of normal. BMI levels of participants in the high increasing group were consistently significantly higher than normal increasing group at any age points, and this difference can be seen as early as childhood. In the resolving group, BMI increased more rapidly than that of the high increasing group, resulting in an obesity status in childhood (8-10 years), but it dropped to the normal level in puberty (12-18 years). The trajectory parameters were all significantly different from $0 \quad(\mathrm{p}<0.05)$. The curve parameters of fixed and random effects in the three trajectory groups are shown in online supplemental table 5. BMI trajectories in men and women were similar (online supplemental figure 2).

Table 2 summarises the characteristics of study variables by BMI trajectory groups. Significant differences

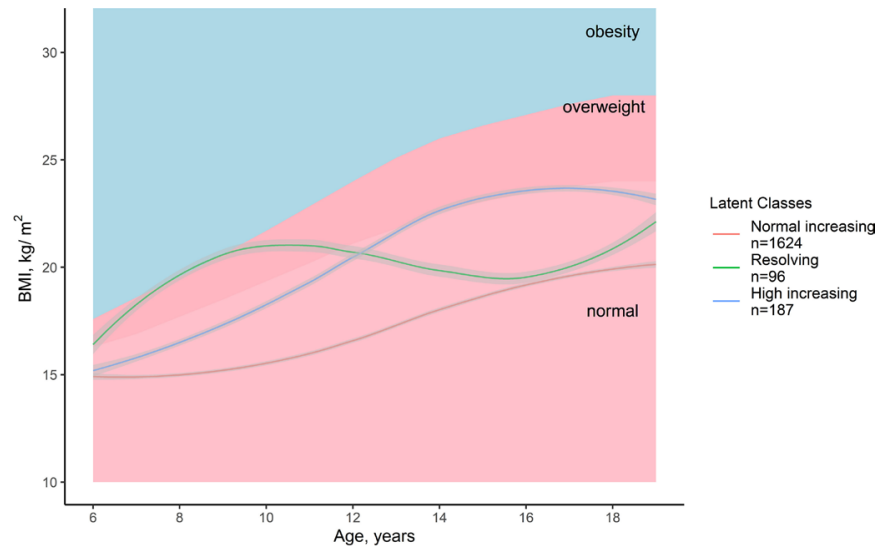

Figure 1 Predicted trajectories of BMI from childhood to puberty. The trajectories are shown in solid lines, and the 95\% Cls are shown in shadow. Overweight and obesity were defined by the health industry standard of the People's Republic of China (WS/T 586-2018) issued by National Health Commission of the People's Republic of China. See detailed information on the curve parameters in online supplemental table 5. BMI, body mass index. 
Table 2 Baseline and follow-up characteristics by latent BMI pattern classes

\begin{tabular}{|c|c|c|c|c|}
\hline Variable & $\begin{array}{l}\text { Normal increasing } \\
1624(85.2 \%)\end{array}$ & $\begin{array}{l}\text { Resolving } \\
96(5.0 \%)\end{array}$ & $\begin{array}{l}\text { High increasing } \\
187(9.8 \%)\end{array}$ & $\mathrm{P}$ value \\
\hline \multicolumn{5}{|l|}{ Baseline } \\
\hline Male, n (\%) & $882(54.3)$ & $45(46.8)$ & $100(53.4)$ & 0.363 \\
\hline Urban, n (\%) & $422(25.9)$ & $30(31.2)$ & $54(28.8)$ & 0.392 \\
\hline $\mathrm{SBP}, \mathrm{mm} \mathrm{Hg}{ }^{*}$ & $88.7(10.1)$ & $90.4(13.1)$ & $91.3(10.1)$ & 0.001 \\
\hline DBP, $\mathrm{mm} \mathrm{Hg}^{*}$ & $57.8(8.3)$ & $59.4(9.4)$ & $60.6(7.6)$ & $<0.001$ \\
\hline \multicolumn{5}{|l|}{ Follow-up } \\
\hline Age, years & $16.0(2.0)$ & $15.8(1.9)$ & $15.6(2.1)$ & 0.308 \\
\hline DBP, mm Hg* & $69.4(8.9)$ & $71.5(8.0)$ & $72.0(8.0)$ & $<0.001$ \\
\hline Follow-up, years & $7.3(1.8)$ & $7.5(2.0)$ & $7.1(1.6)$ & 0.393 \\
\hline HBP, n (\%) & $142(8.7)$ & $8(8.3)$ & $29(15.5)$ & 0.010 \\
\hline
\end{tabular}

Data are means (SD) or $\mathrm{n}(\%)$.

$P$ values: the differences between normal increasing group, resolving group and high increasing group.

${ }^{*}$ Results of SBP/DBP were calculated after removing missing value

BMI, body mass index; DBP, diastolic blood pressure; HBP, high blood pressure; SBP, systolic blood pressure.

in BMI, SBP and DBP among the three trajectory groups were observed at baseline $(\mathrm{p}<0.001)$. The high increasing group had the highest follow-up BMI of 23.5 $\mathrm{kg} / \mathrm{m}^{2}$, followed by the resolving group, and the normal increasing group had the lowest follow-up BMI of 19.0 $\mathrm{kg} / \mathrm{m}^{2}$, and the differences were statistically significant $(\mathrm{p}<0.001)$.
Table 3 presents HRs and 95\% CIs for the association between the trajectory groups and HBP. Compared with the reference (normal increasing) group, the unadjusted HRs (95\% CIs) for the resolving and high increasing groups were 0.91 (0.45 to 1.86$)$ and 1.88 (1.26 to 2.81), respectively. After adjusting for baseline age, region, sex and baseline BMI z-score in model 2, the HRs (95\% CIs) for the resolving and

Table $3 \mathrm{HRs}$ and $95 \% \mathrm{Cls}$ of BMI trajectory groups for high blood pressure

\begin{tabular}{|c|c|c|c|c|c|c|}
\hline & \multicolumn{2}{|l|}{ Model 1} & \multicolumn{2}{|l|}{ Model 2} & \multicolumn{2}{|l|}{ Model 3} \\
\hline & HR (95\% Cl) & $P$ value & HR (95\% Cl) & $P$ value & HR (95\% Cl) & $P$ value \\
\hline \multicolumn{7}{|l|}{ Total } \\
\hline Resolving & 0.91 (0.45 to 1.86$)$ & 0.799 & 0.67 (0.30 to 1.47$)$ & 0.317 & 0.66 (0.30 to 1.45$)$ & 0.306 \\
\hline High increasing & 1.88 (1.26 to 2.81 ) & 0.002 & 1.66 (1.09 to 2.54$)$ & 0.018 & 1.56 (1.02 to 2.38$)$ & 0.040 \\
\hline Resolving & 0.88 (0.32 to 2.41$)$ & 0.799 & 0.53 (0.18 to 1.57$)$ & 0.252 & 0.54 (0.19 to 1.59$)$ & 0.266 \\
\hline High increasing & 2.33 (1.32 to 4.11$)$ & 0.003 & 2.05 (1.14 to 3.72 ) & 0.017 & 1.90 (1.05 to 3.45$)$ & 0.035 \\
\hline \multicolumn{7}{|l|}{ Male } \\
\hline Normal increasing & 1.00 (reference) & & 1.00 (reference) & & 1.00 (reference) & \\
\hline
\end{tabular}

Model 1: Unadjusted for any covariates

Model 2: Adjusted for baseline age, region, sex (only for total) and baseline BMI z-score

Model 3: Adjusted for baseline age, region, sex (only for total), baseline BMI z-score, baseline SBP and baseline DBP

BMI, body mass index; DBP, diastolic blood pressure; SBP, systolic blood pressure. 


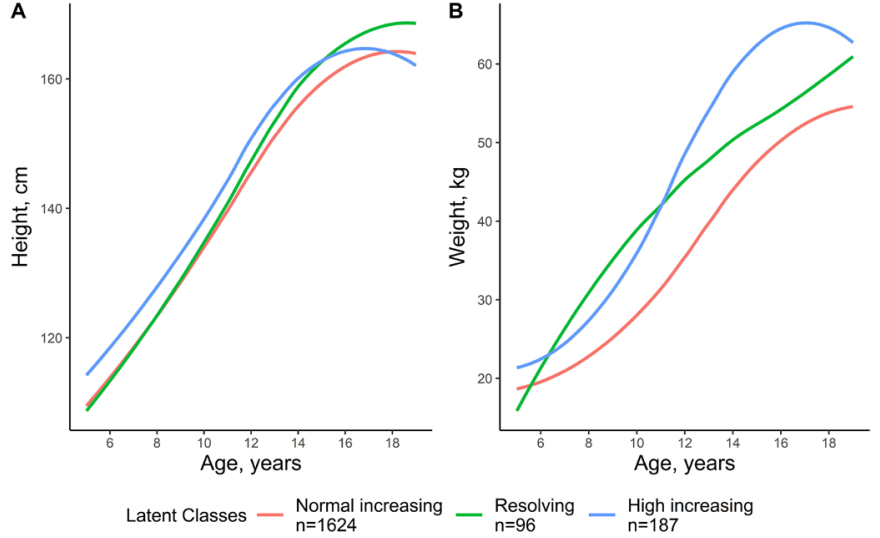

Figure 2 Trajectories of height and weight from childhood to puberty. (A) for height; (B) for weight.

high increasing groups were 0.67 (0.30 to 1.47 ) and 1.66 (1.09 to 2.54), respectively. After additional adjustment for baseline SBP level and baseline DBP level in model 3, the HRs (95\% CIs) were 0.66 (0.30 to 1.45 ) and 1.56 (1.02 to 2.38). In addition, we found similar relationship between the BMI trajectory groups and HBP in male and female respondents, except that the HR (95\% CI) for the resolving group was $1.02(0.37$ to 2.78$)$ without any adjustment in model 1 among male respondents. Online supplemental table 6 presents $\beta$ and SE for the linear regression used to analyse the associations between BMI trajectory groups and SBP/DBP. The results of linear regression showed the same trend as the Cox proportional hazard model. Compared with the reference (normal increasing) group, the high increasing group was associated with a higher level of SBP $(\beta=3.44, p<0.001)$, and similar pattern was also observed in DBP $(\beta=1.65$, $\mathrm{p}=0.024$ ) with adjusting for baseline age, region, follow-up years, sex and baseline BMI z-score, baseline SBP and baseline DBP in model 3. There was no significant association between the resolving group and SBP/DBP, except that it was associated with a higher level of DBP $(\beta=2.11, p=0.033)$ without any adjustment in model 1 .

Figure 2 shows the trajectories of height and weight change in this study cohort. At puberty, the resolving group showed a higher rate of height change than the high increasing group, although the height level was slightly lower. Even at ages 16-18, the resolving group had a higher height level than the high increasing group. In contrast, for the weight, the high increasing group had a considerably higher increasing rate and much higher weight level than the resolving group. Online supplemental figure 3 shows the changes of nutrition intake in different trajectory groups. Energy and fat intake in the high increasing group increased significantly and remained high throughout the period of puberty (10-16 years).

\section{DISCUSSION}

In this community-based cohort with repeated measurements of BMI, we characterised BMI growth trajectories from childhood to puberty and explored the relationship between BMI trajectory groups and HBP incidence. We observed three distinct trajectories of BMI from childhood to puberty that were associated differently with the risk of HBP. Previous observational studies have shown that the BMI levels during adolescence can influence the incidence of hypertension. ${ }^{5-8}$ But this study is one of the first studies to explore the trajectories of BMI in Chinese children and adolescents and their relationship with HBP. This study provides new insights into the origins of obesity-related HBP in childhood and adolescence and highlights the importance of BMI trajectories during adolescence in assessing the risk of HBP.

Of three different BMI growth trajectories found in this study, normal increasing group $(85.16 \%, \mathrm{n}=1624)$ accounted for the majority, in which BMI remained a low level within normal weight status throughout follow-up. In the high increasing group, BMI increased gradually, reaching a peak around age 16 and exceeding the upper limit of normal. However, compared with the resolving group, the high increasing group had a lower baseline BMI z-score and remained within the normal range at follow-up. Compared with the normal increasing group, the HR (95\% CI) for the high increasing was 1.88 (1.26 to 2.81). Ahanchi $e t a l^{23}$ previously reported that among women, trend of increasing BMI parallel with age can be a better predictor for risk of developing hypertension than those with higher BMI at baseline. Börnhorst $e t a l^{24}$ reported the conclusion that higher BMI growth during all periods was related to a higher metabolic risk independent of prior BMI growth, BMI at birth and confounding factors. Fan $e t a l^{25}$ reported that the level-independent BMI trajectories during young adulthood have significant impact on hypertension risk. All of the studies mentioned above came to similar conclusions to the current study, although we focused on different ages.

Remarkably, we found a resolving group $(5.03 \%, \mathrm{n}=96)$ in which BMI increased rapidly than that of the high increasing group, resulting in an obesity status in childhood (8-10 years), but it dropped to normal level in puberty (12-18 years). Our Cox regression analyses showed that the resolving group had a lower risk of HBP than the normal increasing group, with a $\mathrm{HR}$ value of 0.67 though not statistically significant. There are limited comparable literatures with similar research objectives. Buscot $e t a l^{15}$ reported a resolving group in which participants were affected by overweight or obesity in childhood but progressively reversed their elevated BMI status between 30 and 50 years of age. For this group, the risk ratio of HBP was 0.76 compared with the stable normal group. Norris et $a l^{26}$ developed a growth mixture model to capture heterogeneity in BMI trajectories between 7.5 and 24.5 years and found that individuals with high BMI across childhood had lower cardiometabolic disease risk than those who did not become overweight or obese until late adolescence. Ohlsson $e t a l^{27}$ reported that boys who were overweight during puberty and those who remained overweight throughout childhood and puberty, but not those who were overweight during childhood but returned to normal during puberty, had an increased risk of cardiovascular mortality. These conclusions are consistent 
with our conclusion that controlling BMI during puberty can significantly reduce the risk of HBP and highlight that puberty is a crucial period for the development of HBP. As emphasised by the critical period model, the timing of exposure such as at a specific time in the life process which can have a lasting effect on anatomy or physiological function that may ultimately lead to disease. ${ }^{28}$ Individuals with obesity have lower levels of growth hormone. This reduction is functional and can be almost reversed by weight loss but can lead to anthropometric and metabolic changes that increase cardiometabolic risk. ${ }^{29}$ In addition, obesity-induced insulin resistance in adolescents with obesity may also have a relationship with endothelial dysfunction and increased carotid intima-media thickness. ${ }^{3031}$ Our findings suggest that reversing high BMI levels and BMI change rate in puberty may lead to better blood pressure profiles than remaining stable high increasing.

In addition, we found that energy and fat intake in the high increasing group increase significantly and remained high throughout the period of puberty (10-16 years). Ambrosini et $a l^{32}$ reported that an energy-dense, high-fat diet pattern might be positively associated with obesity. Several studies explored the relationship between dietary patterns and cardiovascular disease using data from CHNS and drew the conclusion that modern dietary patterns with high energy and high fat intake were associated with a higher risk of adverse cardiovascular disease (CVD) outcomes. ${ }^{33-35}$ To sum up, it is of great significance to adjust the dietary pattern in puberty and reduce the intake of energy and fat for reducing the increasing level of BMI and the incidence of HBP.

\section{Comparison with previous studies}

The current study is one of the first studies to focus on the BMI trajectories from children to adolescence. Recently, Wang et al ${ }^{36}$ assessed the BMI trajectoryrelated hypertension risk with the data of Zhongshan school physical examination database. Ziyab $e^{119} l^{19}$ identified trajectories of BMI from 1 to 18 years of age and determined whether BMI trajectories predict blood pressure at 18 years. But our study has some additional advantages. First, group-based trajectory modelling (GBTM) was applied in both of the studies mentioned above. GBTM is a device to estimate trajectory distribution under the premise of unknown population distribution. The trajectory groups should be taken as clusters of individuals following the same trajectory approximately. But LCGMM was used in our study, so that we could find more personalised growth trajectories that actually exist. Second, we identified a group that returned to normal BMI increasing levels that could be used to compare outcomes between participants who had controlled the BMI increase trend during adolescence and those who remained highly increased. Third, we fitted changes in nutrient intake, which are the important risk factors for HBP, as well as the trends in height and weight of the participants to analyse the possible causes and processes of the BMI trajectories.

\section{Strengths and weaknesses}

This study had several strengths. The cohort from which we extracted the data is an Asian population with a relatively large cohort size, long follow-up years and repeated measures of study variables over time using a consistent study protocol. What is more, the LCGMM approach provides a posteriori identification of distinct BMI trajectories, thus avoiding misclassification and loss of information. In addition, we used incidence instead of prevalence, which is suitable for analysing the HBP levels in different BMI trajectory groups in specific population. On the other hand, several limitations of this study also need to be considered. First, the assessment of HBP was partly based on the self-reports. The incidence of HBP may be underestimated in CHNS, which may lead to the bias of Cox proportional hazard model results. Second, compared with the children included in the study, a large proportion of children were excluded, and there were significant differences between the two groups, which may lead to selection bias. Third, due to the lack of relevant data in CHNS, biological maturity indicators have not been adjusted, which may affect the estimation of the impact of BMI trajectories on blood pressure.

\section{CONCLUSIONS}

In summary, the current study identified three distinct trajectories of BMI from childhood to puberty for Chinese. The high increasing group had a significantly high risk of HBP. However, for the resolving group in which BMI dropped to the normal level in puberty there was no significant impact on the risk of HBP. Puberty is a crucial period for the development of HBP. Early prevention and intervention by adjusting dietary patterns in puberty are of great significance to prevent HBP. This study provides new insights into the origins of obesity-related HBP in childhood and adolescence and highlights the importance of BMI trajectories during adolescence in assessing the risk of HBP, although further detailed studies with large sample sizes are needed to support our results.

Acknowledgements This research uses data from China Health and Nutrition Survey (CHNS). We thank the National Institute of Nutrition and Food Safety, China Center for Disease Control and Prevention, Carolina Population Center, the University of North Carolina at Chapel Hill, the NIH (R01-HD30880, DK056350 and R01-HD38700) and the Fogarty International Center, NIH for financial support for the CHNS data collection and analysis files from 1989 to 2006 and both parties plus the China-Japan Friendship Hospital, Ministry of Health for support for CHNS 2009 and future surveys.

Contributors All authors had played a role in study concept and design (YJ, YuL and XZ); YuL is responsible for the overall content as a guarantor; acquisition of data (YuL); analysis and interpretation of data (YJ, SW and YW); statistical analysis (YQ, YiL and YF); drafting of the manuscript (YJ); critical revision of the manuscript for important intellectual content and study supervision (YuL, XZ and FX).

Funding This study was supported by grant from National Natural Science Foundation of China (81973147), Youth Team of Humanistic and Social Science of Shandong University (20820IFYT1902), Cheeloo Young Scholars Program of Shandong University, Shandong University multidisciplinary research and innovation team of young scholars (2020QNQT11 and IFYT18034).

Competing interests None declared. 
Patient consent for publication Not applicable.

Ethics approval The protocol was approved by the Ethics Committee of the National Institute for Nutrition and Health, China CDC (Number 201524). Informed consent was obtained from all subjects before the investigation.

Provenance and peer review Not commissioned; externally peer reviewed.

Data availability statement Data are available in a public, open access repository. The data for this study were from the China Health and Nutrition Survey (CHNS) database, accessed openly to https://www.cpc.unc.edu/projects/china/data.https:// www.cpc.unc.edu/projects/china/data.

Supplemental material This content has been supplied by the author(s). It has not been vetted by BMJ Publishing Group Limited (BMJ) and may not have been peer-reviewed. Any opinions or recommendations discussed are solely those of the author(s) and are not endorsed by BMJ. BMJ disclaims all liability and responsibility arising from any reliance placed on the content. Where the content includes any translated material, BMJ does not warrant the accuracy and reliability of the translations (including but not limited to local regulations, clinical guidelines, terminology, drug names and drug dosages), and is not responsible for any error and/or omissions arising from translation and adaptation or otherwise.

Open access This is an open access article distributed in accordance with the Creative Commons Attribution Non Commercial (CC BY-NC 4.0) license, which permits others to distribute, remix, adapt, build upon this work non-commercially, and license their derivative works on different terms, provided the original work is properly cited, appropriate credit is given, any changes made indicated, and the use is non-commercial. See: http://creativecommons.org/licenses/by-nc/4.0/.

ORCID iDs

Yiman Ji http://orcid.org/0000-0001-8554-7221

Yunxia Liu http://orcid.org/0000-0002-0388-1195

\section{REFERENCES}

1 Wang Z, Chen Z, Zhang L, et al. Status of hypertension in China: results from the China hypertension survey, 2012-2015. Circulation 2018;137:2344-56.

2 Fang L, Song J, Ma Z, et al. Prevalence and characteristics of hypertension in mainland Chinese adults over decades: a systematic review. J Hum Hypertens 2014;28:649-56.

3 Anyaegbu El, Dharnidharka VR. Hypertension in the teenager. Pediatr Clin North Am 2014;61:131-51.

4 Wühl E. Hypertension in childhood obesity. Acta Paediatr 2019;108:37-43.

5 Dong B, Ma J, Wang HJ, et al. The association of overweight and obesity with blood pressure among Chinese children and adolescents. Biomed Environ Sci 2013;26:437-44.

6 Cai L, Wu Y, Wilson RF, et al. Effect of childhood obesity prevention programs on blood pressure: a systematic review and meta-analysis. Circulation 2014;129:1832-9.

7 Dong Y, Jan C, Zou Z, et al. Effect of overweight and obesity on high blood pressure in Chinese children and adolescents. Obesity 2019;27:1503-12.

8 Yang Y, Dong B, Wang S, et al. Prevalence of high blood pressure subtypes and its associations with BMI in Chinese children: a national cross-sectional survey. BMC Public Health 2017;17:598.

9 Tu AW, Mâsse LC, Lear SA, et al. Body mass index trajectories from ages 1 to 20: results from two nationally representative Canadian longitudinal cohorts. Obesity 2015;23:1703-11.

10 Ventura AK, Loken E, Birch LL. Developmental trajectories of girls' BMI across childhood and adolescence. Obesity 2009;17:2067-74.

11 Magee CA, Caputi P, Iverson DC. Identification of distinct body mass index trajectories in Australian children. Pediatr Obes 2013;8:189-98.

12 Zhang T, Whelton PK, Xi B, et al. Rate of change in body mass index at different ages during childhood and adult obesity risk. Pediatr Obes 2019;14:e12513.

13 Evensen E, Wilsgaard T, Furberg A-S, et al. Tracking of overweight and obesity from early childhood to adolescence in a populationbased cohort - the Tromsø Study, Fit Futures. BMC Pediatr 2016;16:64.
14 Li S, Chen W, Sun D, et al. Variability and rapid increase in body mass index during childhood are associated with adult obesity. Int $J$ Epidemiol 2015;44:1943-50.

15 Buscot M-J, Thomson RJ, Juonala M, et al. Distinct child-to-adult body mass index trajectories are associated with different levels of adult cardiometabolic risk. Eur Heart J 2018;39:2263-70.

16 Popkin BM, Du S, Zhai F, et al. Cohort Profile: The China Health and Nutrition Survey--monitoring and understanding socioeconomic and health change in China, 1989-2011. Int J Epidemiol 2010;39:1435-40.

17 Zhang B, Zhai FY, Du SF, et al. The China health and nutrition survey, 1989-2011. Obes Rev 2014;15 Suppl 1:2-7.

18 Writing Group of the 2018 Chinese Guidelines for the Management of Hypertension. 2018 Chinese guidelines for the management of hypertension. Chin J Cardiovasc Med 2019;24:24-56.

19 Ziyab AH, Karmaus W, Kurukulaaratchy RJ, et al. Developmental trajectories of Body Mass Index from infancy to 18 years of age: prenatal determinants and health consequences. J Epidemiol Community Health 2014;68:934-41.

20 Tu Y-K, Tilling K, Sterne JAC, et al. A critical evaluation of statistical approaches to examining the role of growth trajectories in the developmental origins of health and disease. Int $\mathrm{J}$ Epidemiol 2013;42:1327-39.

21 Geserick M, Vogel M, Gausche R, et al. Acceleration of BMI in early childhood and risk of sustained obesity. $N$ Engl J Med 2018;379:1303-12.

22 Proust-Lima C, Philipps V, Liquet B. Estimation of extended mixed models using latent classes and latent processes: the $R$ package Icmm. J Stat Softw 2015;78:1-56.

23 Ahanchi NS, Ramezankhani A, Munthali RJ, et al. Body mass index trajectories from adolescent to young adult for incident high blood pressure and high plasma glucose. PLoS One 2019;14:e0213828.

24 Börnhorst C, Tilling K, Russo P, et al. Associations between early body mass index trajectories and later metabolic risk factors in European children: the IDEFICS study. Eur J Epidemiol 2016;31:513-25.

25 Fan B, Yang Y, Dayimu A, et al. Body mass index trajectories during young adulthood and incident hypertension: a longitudinal cohort in Chinese population. J Am Heart Assoc 2019;8:e011937.

26 Norris T, Mansukoski L, Gilthorpe MS, et al. Distinct body mass index trajectories to Young-Adulthood obesity and their different cardiometabolic consequences. Arterioscler Thromb Vasc Biol 2021;41:1580-93.

27 Ohlsson C, Bygdell M, Sondén A, et al. Association between excessive BMI increase during puberty and risk of cardiovascular mortality in adult men: a population-based cohort study. Lancet Diabetes Endocrinol 2016;4:1017-24.

28 Lynch J, Smith GD. A life course approach to chronic disease epidemiology. Annu Rev Public Health 2005;26:1-35.

29 Savastano S, Di Somma C, Barrea L, et al. The complex relationship between obesity and the somatropic axis: the long and winding road. Growth Horm IGF Res 2014;24:221-6.

30 Ruiz LD, Zuelch ML, Dimitratos SM, et al. Adolescent obesity: diet quality, psychosocial health, and cardiometabolic risk factors. Nutrients 2019;12:43.

31 Beauloye V, Zech F, Tran HTM, et al. Determinants of early atherosclerosis in obese children and adolescents. J Clin Endocrinol Metab 2007;92:3025-32.

32 Ambrosini GL, Emmett PM, Northstone K, et al. Tracking a dietary pattern associated with increased adiposity in childhood and adolescence. Obesity 2014;22:458-65.

33 Lee Y-H, Shelley M, Liu C-T, et al. Assessing the association of food preferences and self-reported psychological well-being among middle-aged and older adults in contemporary China-Results from the China health and nutrition survey. Int $J$ Environ Res Public Health 2018;15:463.

34 Zhen S, Ma Y, Zhao Z, et al. Dietary pattern is associated with obesity in Chinese children and adolescents: data from China health and nutrition survey (CHNS). Nutr J 2018;17:68.

35 Li M, Shi Z. Dietary pattern during 1991-2011 and its association with cardio metabolic risks in Chinese adults: the China health and nutrition survey. Nutrients 2017;9:1218.

36 Wang $X$, Dong B, Huang S, et al. Body mass index trajectory and incident hypertension: results from a longitudinal cohort of Chinese children and adolescents, 2006-2016. Am J Public Health 2020;110:1689-95. 